\title{
Staphylococcus Aureus Carriage in French Athletes at Risk of CA-MRSA Infection: a Prospective, Cross-sectional Study
}

\author{
E. Couvé-Deacon 1,2,3 , D. Postil ${ }^{4}$, O. Barraud ${ }^{1,2,3}$, C. Duchiron ${ }^{4}$, D. Chainier ${ }^{1,2,3}$, A. Labrunie ${ }^{5}$, N. Pestourie ${ }^{3}$, P.M. Preux ${ }^{5}$, \\ B. François ${ }^{1,2,4}$ and M.C. Ploy ${ }^{1,2,3^{*}}$
}

\begin{abstract}
Background: Staphylococcus aureus (SA) is a leading cause of infectious diseases in sports teams. In recent decades, community-associated SA (CA-SA) strains have emerged worldwide and have been responsible for outbreaks in sports teams. There are very few data on the prevalence of these strains in France, and none on the carriage among athletes.

Methods: We conducted a cross-sectional study to determine the SA carriage proportion among athletes practicing sports at risk for CA-SA infection in a French county, and determined the methicillin-resistant and/or CA-SA proportion. We also analyzed SA carriage according to risks factors and studied the SA clonality in a sample of our population.

Results: We included 300 athletes; SA carriage proportion was 61\% $(n=183)$ and one was MRSA carrier $(0.33 \%)$. The MRSA strain belonged to the clonal complex ST5. None of the strain produced Panton Valentine Leucocidin, and we did not find clonal distribution within the teams. Interestingly, we found a high throat-only carriage $(n=57), 31.1 \%$ of the SA carriers.
\end{abstract}

Conclusion: We found a high SA carriage with a local epidemiology quite different than that reported in a similar population in the USA. Further studies on SA carriage should include throat sampling.

Trial registration: The approved protocol was registered on ClinicalTrial.gov, NCT01148485.

Keywords: Staphylococcus aureus, Community associated, Panton Valentine leukocidin, Nasal carriage, Throat carriage, Sports team

\section{Keypoints}

- The prevalence of $S$. aureus carriage in at-risk athletes was high but MRSA carriage was low and there was no community associated SA carriage.

- We found no clear evidence of clonal transmission. SA carriage out of outbreak period is probably complex, resulting of a balance between host susceptibility and endogenous flora with skin contact transmission within a sports team.

- Throat-only carriage represented a high percentage of the SA carriers which substantially inflated the

\footnotetext{
* Correspondence: marie-cecile.ploy@unilim.fr

'University Limoges, UMR, 1092 Limoges, France

${ }^{2}$ INSERM, UMR, 1092 Limoges, France

Full list of author information is available at the end of the article
}

nose carriage. Studies on SA carriage should include throat sampling for a better accuracy.

- Staphylococcal carriage prevention in this population could consist of simple hygiene measures promotion, known to efficiently halt SA transmission in sports teams.

\section{Background}

Staphylococcus aureus (SA) is a leading cause of both community-acquired and healthcare-associated infections. Community-associated methicillin-resistant S. aureus (CA-MRSA) emerged worldwide during the last decade. CA-MRSA causes skin and soft-tissue infections such as multiple abscesses among healthy individuals, as well as life-threatening necrotizing pneumonia in children and young adults [1]. There is a strong epidemiological link 
between CA-MRSA causing deep primary skin infections and Panton-Valentine leukocidin (PVL). PVL-producing SA strains have a peculiar antibiotic susceptibility profile and are more susceptible to antimicrobials as compared to healthcare-associated MRSA [2]. The prevalence of CAMRSA seems to be low but is increasing in Europe, although prevalence in clinical isolates vary according to the country from less than 0.5 to $15 \%$ across published studies [3].

Skin-to-skin contact and poor hygiene are risk factors for CA-MRSA outbreaks, and populations at risk of CAMRSA infection include prisoners, military personnel, and sports teams [4]. Most studies of SA infections among athletes have been conducted in the USA, and also in the UK, Germany, and Japan $[5,6]$. In the USA, CA-MRSA infections among athletes have become very frequent, the most at-risk sports being those involving physical contact, such as American football, rugby, and wrestling $[5,6]$. CA-MRSA infections have also been linked to sports involving less physical contact but shared equipment, such as fencing, martial arts, crosscountry running, volleyball, basketball, football, baseball, and weight-lifting [5]. Other sport-related SA infection risk factors include a high body mass index, use of equipment resulting in skin abrasion, and poor personal hygiene (sharing of personal items, failure to protect skin lesions) [6].

The anterior nasal cavities are the most common SA carriage site with the oropharynx (10-50\%), but skin carriage is also frequent, especially on the hands (27-90\%), perineum (22-60\%), and axilla (8-19\%), the intestinal tract, vagina, and skin lesions are also common carriage sites [7]. Nasal carriage has been associated in literature with a higher incidence of SA infections [7]. Twenty to $25 \%$ of healthy volunteers were reported to be permanent nasal carriers, $60 \%$ intermittent carriers and $20 \%$ permanent non-carriers [8]. In the US general population, $1.3 \%$ were nasal MRSA carriers, compared to $5.4 \%$ of athletes and individuals in daily contact with MRSA carriers $[9,10]$.

In France, the reported SA nasal carriage rates were about one third of the general population. However, unlike the USA, there are very few data on the prevalence of CA-MRSA in France, and none on carriage among athletes at risk of CA-MRSA infection. The aim of this study was to determine the SA carriage proportion among athletes practicing physical contact sports in a French county, and the proportion and ST types of isolates resistant to methicillin and/or producing the PVL. We also analyzed SA carriage according to the sport, hygiene habits, and medical history.

\section{Methods}

\section{Study Design}

We conducted a cross-sectional study of a representative sample of athletes in the French county of Limousin.
We selected sports at risk of CA-MRSA infection, namely rugby, wrestling, basketball, volleyball, handball, fencing, martial arts, football, weight-lifting and baseball.

Based on the reported SA nasal carriage of 27 to $37.2 \%$ in the general population but with previous rate reported around $35 \%$ in the literature $(15,29)$, we determined that the required population size for an expected SA carriage proportion of $35 \%$, a precision of $8 \%$, and a type 1 risk of 5\% was 150 subjects, calculated using Nquery Advisor 7.0 (Statistical Solutions, Saugus, MA, USA). The estimated sample size anticipated a missing data rate of $10 \%$. We used cluster sampling-the cluster being a sports team-that included all registered teams practicing the targeted sports in Limousin. The teams were randomly selected. All subjects in a selected team were eligible for enrollment. As cluster sampling doubles the required population size, we included 300 subjects.

The study, as biomedical research, was authorized by the French competent authorities and the local ethics committee (CPP). The study was carried out exactly as described in the approved protocol (Clinicaltrials.gov, NCT01148485). The study was proposed to the randomly selected sport teams. When we obtained the team agreement, an appointment during a training session was decided. During the training session, participation was proposed to all present athletes. After information and obtainment of the signed consent of the athletes, the study sampling was done.

\section{Inclusion and Exclusion Criteria}

The inclusion criteria were age over 18 years, registration in a Limousin sport club, and practice of at least one of the selected sports. Exclusion criteria were inability to complete the study questionnaire or if sampling was not possible. Each participant signed informed consent forms and samples were collected during regular training sessions.

The athletes completed a questionnaire on demographic characteristics, sport practice, personal hygiene, and medical history.

\section{Microbiological Procedures}

Swab samples of the nose, throat, groin and skin lesions (defined as excoriation, erosion or crust of the skin) were transported to the microbiological laboratory at ambient temperature, seeded on $S$. aureus specific chromogenic media (chromID ${ }^{\mathrm{tm}}$ S. aureus, bioMerieux, Lyon, France) and incubated for $48 \mathrm{~h}$ at $37{ }^{\circ} \mathrm{C}$ in an aerobic atmosphere. SA was identified on the basis of colony aspect, Gram staining, and catalase, and coagulase production (Pastorex ${ }^{\oplus}$, Fumouze, Levallois-Perret, France).

The antibiotic susceptibility profile of isolates was determined as recommended in the guidelines of the Antibiogram Committee of the French Microbiology Society 
(CA-SFM). Methicillin susceptibility was studied with cefoxitin disks. When this failed to categorize the isolate as susceptible or resistant, the mecA gene (encoding methicillin resistance) was sought by real-time PCR with specific primers, the method was adapted from a previously published protocol [11]. The PVL-encoding gene was also sought by PCR in all SA isolates, as previously described [12]. MRSA strains were characterized by Multi-Locus Sequence Typing, Enright et al. method [13]. MLST is based on the sequencing of seven housekeeping genes, defining an allelic profile corresponding to a Sequence Type (ST). ST designations were those assigned by the MLST database (available from: URL: http://www.mlst.net).
An athlete was defined as a carrier if at least one strain of $S$. aureus was isolated from at least one of his or her samples, and as an MRSA carrier if at least one SA strain resistant to methicillin was detected in at least one sample.

\section{ERIC PCR Strain Typing}

We performed ERIC PCR for strain molecular typing [14] with the ERIC2 primer. To study clonal transmission within each team and between the different teams, we choose a sample of the strains isolated. Amplification products were separated by agarose gel electrophoresis in $2.0 \%$ agarose, and visualized by u.v. transillumination. The fingerprints obtained by ERIC-PCR were visually

Table 1 General Description of the Population

\begin{tabular}{|c|c|c|c|c|c|c|c|}
\hline Label & Mean & Std Dev & Minimum & Maximum & Lower quartile & Median & Upper quartile \\
\hline Age & 30.40 & 10.10 & 18.02 & 86.77 & 22.76 & 27.97 & 35.69 \\
\hline Height & 176.04 & 7.75 & 154.00 & 200.00 & 170.00 & 176.00 & 181.00 \\
\hline Weight & 76.38 & 13.44 & 44.00 & 140.00 & 67.00 & 76.00 & 85.00 \\
\hline Body Mass Index & 24.53 & 3.52 & 17.20 & 43.20 & 22.10 & 24.20 & 26.10 \\
\hline variable & \multicolumn{3}{|c|}{ Modality } & \multicolumn{2}{|c|}{ Number of subjects } & \multicolumn{2}{|l|}{ Percent } \\
\hline \multirow[t]{2}{*}{ Gender } & \multicolumn{3}{|l|}{ M } & \multicolumn{2}{|l|}{247} & \multicolumn{2}{|l|}{82.33} \\
\hline & \multicolumn{3}{|l|}{$\mathrm{F}$} & \multicolumn{2}{|l|}{53} & \multicolumn{2}{|l|}{17.67} \\
\hline \multirow[t]{2}{*}{ linked to healthcare system profession } & \multicolumn{3}{|l|}{ NO } & \multicolumn{2}{|l|}{283} & \multicolumn{2}{|l|}{95.93} \\
\hline & \multicolumn{3}{|l|}{ YES } & \multicolumn{2}{|l|}{12} & \multicolumn{2}{|l|}{4.07} \\
\hline \multirow[t]{4}{*}{ Sport competition level ${ }^{a}$} & \multicolumn{3}{|c|}{ National } & \multicolumn{2}{|l|}{31} & \multicolumn{2}{|l|}{13.6} \\
\hline & \multicolumn{3}{|c|}{ Pre-National } & \multicolumn{2}{|l|}{19} & \multicolumn{2}{|l|}{8.33} \\
\hline & \multicolumn{3}{|l|}{ Region } & \multicolumn{2}{|l|}{142} & \multicolumn{2}{|l|}{62.28} \\
\hline & \multicolumn{3}{|l|}{ Local } & \multicolumn{2}{|l|}{36} & \multicolumn{2}{|l|}{15.79} \\
\hline Professional/amateur & Profess & & & 8 & & 2.67 & \\
\hline & Amate & & & 292 & & 97.33 & \\
\hline Sport 27 teams & Martial & & & 45 & & 15.00 & \\
\hline & Baseba & & & 31 & & 10.33 & \\
\hline & Basketh & & & 19 & & 6.33 & \\
\hline & Footba & & & 48 & & 16.00 & \\
\hline & Weight & & & 16 & & 5.33 & \\
\hline & Handb & & & 54 & & 18.00 & \\
\hline & Wrestli & & & 11 & & 3.67 & \\
\hline & Rugby & & & 56 & & 18.67 & \\
\hline & Volleyb & & & 20 & & 6.67 & \\
\hline Championship & NO & & & 58 & & 19.53 & \\
\hline & YES & & & 239 & & 80.47 & \\
\hline Number of training session per week & Less th & & & 21 & & 7.02 & \\
\hline & 1 & & & 66 & & 22.07 & \\
\hline & 2 & & & 141 & & 47.16 & \\
\hline & 2 and & & & 71 & & 23.75 & \\
\hline
\end{tabular}

${ }^{a}$ This variable does not include all the sports. Sports as martial arts, wrestling, and weight lifting are not included 
compared. We classified strains in major clones (clone $\mathrm{A}$, clone $\mathrm{B}$, clone $\mathrm{C} . .$.$) that differ by two and more bands$ or minor clones profiles when there was only 1 or 2 bands difference (A1, A2, A3...).

\section{Statistical Analysis}

The study database was created with Oracle-based CLINSIGHT software ( https://ecrf.clinsight.fr/nepha/), notably using the CS-DESIGNER module.

Statistical analysis used SAS V9.2 software (SAS Institute Cary, $\mathrm{NC}$ ), and a $p$ value $<0.05$ was considered to signify statistical significance. Quantitative variables are described as the mean \pm standard deviation (SD) or the median and interquartile interval. Qualitative variables are described as numbers, percentages, and 95\% confidence intervals.

Carriage proportions were compared according the sport and risk factor by using the $\mathrm{chi}^{2}$ test or Fisher's exact test, depending on the theoretical number. To adjust the results, we performed a multivariate logistic regression. We selected the explanatory variables with a $p$ value under 0.25 . The selected variables were then computed to obtain the final model, with the variable to explain being the presence or absence of SA strain in the different samples.

\section{Results}

\section{Population Characteristics}

Between 11 January 2011 and 10 June 2011, we studied 300 athletes, comprising 247 men (82.3\%) and 53 women $(17.7 \%)$. Average age was 30.4 years $( \pm 10.1)$, average height $176.0 \mathrm{~cm}( \pm 7.7)$, average weight $76.4 \mathrm{~kg}$ $( \pm 13.4)$, and the average body mass index $24.5( \pm 3.5)$. The athletes performed at local $(12.0 \%, n=36)$, regional $(47.3 \%, n=142)$, pre-national $(6.3 \%, n=19)$, or national level $(10.3 \%, n=31)$ and these level did not apply for 72 athletes due to their sport. Only one of the 27 studied teams was professional, representing $2.7 \%$ of the 300 athletes (Table 1).

\section{SA and MRSA Carriage}

Among the 300 athletes, $61.0 \%(n=183)$ carried SA $(95 \%$ confidence interval $(95 \% \mathrm{CI})=[51.0 ; 70.0])$. The proportion of MRSA carriage was $0.3 \%(n=1)(95 \% \mathrm{CI}=[0.0 ; 1.4])$ overall and $0.5 \%(n=1)(95 \% \mathrm{CI}=[0.0 ; 1.7])$ among SA carriers $(n=183)$. The MRSA strain belonged to the clonal complex ST5 and was isolated in the nose and skin lesion swabs of a handball player. None of the SA isolates produced PVL. We noticed a high variability of team SA carriage, from $0.0 \%$ in two karate teams $(n=10$ and $n=3)$ to $100 \%$ in a wrestling team $(n=11)$ and a baseball team $(n=6)$. A rugby team had the largest number of members $(n=39)$ and presented a SA carriage of $74.4 \%(n=29)$.
Interestingly, throat carriage was more frequent ( $n=143,47.7 \%)$ than nasal carriage $(n=111,37.0 \%)$, and throat-only carriage was frequent $(n=57,19.0 \%)$ in the population. We sampled 21 skin lesions, and skin lesion-only carriage was $1.7 \%(n=5)$. Multiple sampling sites increased the chances of detecting SA carriage: nasal/throat sampling identified $93.4 \%$ of carriers, while nasal/throat/groin and nasal/throat/skin lesion sampling identified $97.3 \%$ of carriers.. SA carriage was detected only in the throat, nose or groin in respectively $31.1 \%$ $(n=57), 12.0 \%(n=22)$, and $3.2 \%(n=8)$ of SA carriers $(n=183)$ (Table 2).

\section{Risk Factors for SA Carriage}

A history of lengthy ( $>4$ weeks) antibiotic therapy $(p=0.035)$, use of saunas $(p=0.093)$, weight-lifting facilities $(p=0.152)$, and a history of abscess in the previous year $(p=0.096)$ had a $p$ value under 0.250 (Table 3 ) and were included in a multivariate logistic regression model. However, after a selection step of variables none of these four variables was significantly linked to SA carriage. No significant difference in SA carriage proportion was observed between the different sports $(p=0.422)$.

\section{ERIC PCR SA Strain Typing}

We selected teams to study SA clonality. We hypothesized that clone circulation within a team would be easier to

Table 2 SA Carriage According to the Sampling Sites

\begin{tabular}{ll}
\hline Location & $\begin{array}{l}\text { Frequencies } \\
\text { (\% of carriers) } \\
(n=183)\end{array}$ \\
\hline Nose & $111(60.6 \%)$ \\
Nose only & $22(12.0 \%)$ \\
Throat & $143(78.1 \%)$ \\
Throat only & $57(31.1 \%)$ \\
Groin & $40(21.8 \%)$ \\
Groin only & $2(3.2 \%)$ \\
Skin lesion & $21(11.5 \%)$ \\
Skin lesion only & $5(1.7 \%)$ \\
Nose and/or throat & $171(93.4 \%)$ \\
Nose and/or groin & $121(66.1 \%)$ \\
Nose and/or skin lesion & $119(65.0 \%)$ \\
Throat and/or groin & $156(85.2 \%)$ \\
Throat and/or skin lesion & $151(82.5 \%)$ \\
Groin and/or skin lesion & $53(29.0 \%)$ \\
Nose and/or throat and/or groin & $178(97.3 \%)$ \\
Nose and/or throat and/or skin lesion & $178(97.3 \%)$ \\
Nose and/or groin and/or skin lesion & $127(69.4 \%)$ \\
Throat and/or groin and/or skin lesion & $161(88.0 \%)$ \\
Nose and/or throat and/or groin and/or skin lesion & $183(100 \%)$ \\
\hline
\end{tabular}


Table 3 Risk Factors of the Athletes

\begin{tabular}{|c|c|c|c|}
\hline \multicolumn{4}{|l|}{ A. Sport } \\
\hline Factor & & SA Carriers/participants (\%) & $p$ value (test) \\
\hline Baseball & & $18 / 31(58.1 \%)$ & $p=0.422\left(\mathrm{chi}^{2}\right)$ \\
\hline Basketball & & $13 / 19(68.4 \%)$ & \\
\hline Football & & $30 / 48(62.5 \%)$ & \\
\hline Weight-lifting & & $8 / 16(50.0 \%)$ & \\
\hline Handball & & $34 / 54(63.0 \%)$ & \\
\hline Rugby & & $39 / 56(70.0 \%)$ & \\
\hline Individual sports/martial arts (wrestling, judo, karate) & & $33 / 56(58.9 \%)$ & \\
\hline Volleyball & & $8 / 20(40.0 \%)$ & \\
\hline \multicolumn{4}{|l|}{ B. Hygiene } \\
\hline Factor & Modality & SA Carriers/participants (\%) & $p$ value (test) \\
\hline \multirow[t]{2}{*}{ Shared equipment } & NO & $39 / 61(63.9 \%)$ & $p=0.566\left(\mathrm{chi}^{2}\right)$ \\
\hline & YES & $142 / 237(59.9 \%)$ & \\
\hline \multirow[t]{2}{*}{ Shared healthcare materials } & NO & $149 / 240(62.1 \%)$ & $p=0.495\left(\mathrm{chi}^{2}\right)$ \\
\hline & YES & $32 / 56(57.1 \%)$ & \\
\hline \multirow[t]{2}{*}{ Shared personal effects } & NO & 108/176 (61.4\%) & $p=0.946\left(\mathrm{chi}^{2}\right)$ \\
\hline & YES & 75/123 (61.0\%) & \\
\hline \multirow[t]{5}{*}{ Skin damage during sport } & Never & $22 / 42(52.4 \%)$ & $p=0.574$ (Fisher) \\
\hline & Rarely & $57 / 94(60.6 \%)$ & \\
\hline & Sometimes & 75/119 (63.0\%) & \\
\hline & Often & $24 / 35(68.6 \%)$ & \\
\hline & Always & $5 / 10(50.0 \%)$ & \\
\hline \multirow[t]{2}{*}{ Sauna use } & NO & $176 / 285(61.5 \%)$ & $p=0.093\left(c h i^{2}\right)$ \\
\hline & YES & $5 / 13(38.5 \%)$ & \\
\hline \multirow[t]{2}{*}{ Use of training pool } & NO & $180 / 296(60.8 \%)$ & $p=1.000$ (Fisher) \\
\hline & YES & $1 / 2(50.0 \%)$ & \\
\hline \multirow[t]{2}{*}{ Weights room use } & NO & $154 / 246(62.6 \%)$ & $p=0.152\left(c h i^{2}\right)$ \\
\hline & YES & 27/52 (51.9\%) & \\
\hline \multirow[t]{2}{*}{ Skin lesion disinfection } & NO & 77/132 (58.3\%) & $p=0.473\left(\mathrm{chi}^{2}\right)$ \\
\hline & YES & $103 / 165(62.4 \%)$ & \\
\hline \multirow[t]{2}{*}{ Protection of skin lesions } & NO & $119 / 198(60.1 \%)$ & $p=0.905\left(\mathrm{chi}^{2}\right)$ \\
\hline & YES & $59 / 97(60.8 \%)$ & \\
\hline \multirow[t]{2}{*}{ Protection of skin lesions during sport } & NO & $87 / 158(61.4 \%)$ & $p=0.767\left(\mathrm{chi}^{2}\right)$ \\
\hline & YES & 83/139 (59.7\%) & \\
\hline \multirow[t]{3}{*}{ Shower/bath frequency } & Every other day & $23 / 31(74.2 \%)$ & $p=0.267\left(\mathrm{chi}^{2}\right)$ \\
\hline & Once a day & $126 / 211(59.7 \%)$ & \\
\hline & Several times a day & $33 / 57(57.9 \%)$ & \\
\hline \multicolumn{4}{|l|}{ C. Medical history in the previous year } \\
\hline Factor & modality & SA Carriers/participants (\%) & $p$ value (test) \\
\hline \multirow[t]{2}{*}{ Hospitalization } & NO & $156 / 257(60.7 \%)$ & $p=0.795\left(\right.$ chis $\left.^{2}\right)$ \\
\hline & YES & $27 / 43(62.8 \%)$ & \\
\hline \multirow[t]{2}{*}{ Superficial skin lesions } & NO & $130 / 215(60.5 \%)$ & $p=0.763\left(\mathrm{chi}^{2}\right)$ \\
\hline & YES & $53 / 85$ (62.4\%) & \\
\hline
\end{tabular}


Table 3 Risk Factors of the Athletes (Continued)

\begin{tabular}{|c|c|c|c|}
\hline \multirow[t]{2}{*}{ Skin abscess } & $\mathrm{NO}$ & $175 / 291(60.1 \%)$ & $p=0.096$ (Fisher) \\
\hline & YES & 8/9 (88.9\%) & \\
\hline \multirow[t]{2}{*}{ Pneumonia } & NO & 181/297 (60.9\%) & $p=1.000$ (Fisher) \\
\hline & YES & $2 / 3(66.7 \%)$ & \\
\hline \multirow[t]{2}{*}{ Antibiotic treatment } & NO & $158 / 256(61.7 \%)$ & $p=0.447\left(\mathrm{chi}^{2}\right)$ \\
\hline & YES & 21/38 (55.3\%) & \\
\hline \multirow[t]{2}{*}{ Long-term antibiotics } & NO & $182 / 294(61.9 \%)$ & $p=0.035$ (Fisher) \\
\hline & YES & $1 / 6(16.7 \%)$ & \\
\hline \multirow[t]{2}{*}{ Chronic disease } & NO & $173 / 281(61.6 \%)$ & $p=0.440\left(\mathrm{chi}^{2}\right)$ \\
\hline & YES & $10 / 19(52.6 \%)$ & \\
\hline
\end{tabular}

$p<0.25$ value are indicated in italics. Data is presented in three parts: A. SA carriage according to sport, B. SA carriage according to hygiene practice, C. SA carriage according to medical history in the previous year

detect in large team or team with the highest carriage. Fifty-nine isolates were typed. These isolates were obtained from 55 athletes in the 2 largest team rugby teams R012 $(n=39)$ and R031 ( $n=17)$, with, respectively, 71.8 and $58.8 \%$ SA carriage and the two highest carriage teams, L001 (wrestling, $n=11$ ) and B004 (baseball, $n=6$ ) with a $100 \%$ SA carriage. We identified six major clones (clone A to clone F) and 11 minor clones profiles (Table 4). Clones A and B were found in all teams and clone $\mathrm{F}$ was found only in team L001. Altogether, the results did not show a clonal distribution of the SA between the teams. Furthermore, within each team, there was no evidence of a clonal transmission.

\section{Discussion}

We observed an overall $S$. aureus carriage proportion of 61\% (95\%CI: 51.0-70.0) among 300 French athletes. A previous European study showed a carriage rate in France of $21.1 \%$ (95\%CI: 17.4-25.4) among 3870 healthy patients [15], but only the nose was sampled. The prevalence of SA carriage found here in our study is higher than that observed in the general population in other studies with multiple sampling sites [16-18]. This high SA carriage among athletes is not surprising, as SA transmission is facilitated by sharing of equipment and skin-to-skin contacts. In addition, athletes are known to have poor personal hygiene during sports, as reflected by reports of CA-MRSA outbreaks in sports teams $[5,6]$. We report a high variability of team SA carriage, from $0.0 \%$ in a karate team to $100 \%$ in a wrestling team and a baseball team. Clonal transmission of SA within sports teams has already been demonstrated as well as the increase of SA isolates in the sweat of SA carriers during sport practice [19]. However, the typing of the strains did not show clear evidence of clonal transmission within the studied teams. Even though skin contact is known as the main transmission risk in SA outbreaks in sports team, the SA carriage out of outbreak period is probably more complex. Host susceptibility and endogenous flora are probably in balance with skin contact transmission within a sports team.

We observed a higher carriage in the throat $(n=143$, $47.7 \%)$ than in the nose $(n=111,37.0 \%)$, as recently reported in the general population worldwide [18, 20, 21]. Interestingly, throat only carriage represented $31.1 \%$ $(n=57)$ of our carriers $(n=183)$, and represented 19\% of the total population $(n=300)$. Nose positivity accounted for $37 \%$ (111/300 subjects), which is comparable with many other studies on nasal SA carriage in the general population. However, when enriched with throat-only carriers, which are often not sought for in other studies, it added 57 cases, thus inflating the prevalence to close to $60 \%$ (168/300 subjects), which is much higher than most other studies. This raises the question as to what is the genuine rate of $S$. aureus carriage in the general population if throat swabbing would also be performed and highlights the fact that $S$. aureus does not only colonize the nose [16-18]. Due to this peculiar observation we could not really compare our high carriage proportion to other studies. This implies that a combination of throat and nose sampling should be used to detect SA carriage.

In the USA, CA-MRSA is endemic. However, only one publication reported no difference in CA-MRSA nasal carriage between college athletes $(1.8 \%)$ and the general population (1.5\%) [22]. Based on these reports, we expected to find a CA-MRSA carriage proportion of $1.0-2.0 \%$ among French athletes. However, in our study, none carried PVL producing MRSA.

One handball player carried a PVL-negative MRSA, in the nose and skin lesion, the strain belonged to the ST5, the second more frequent clonal complex isolated in France after ST8 [23]. In athletes, MRSA carriage differed according to the studies. A study reported that $23.1 \%$ ( $n=44 / 190)$ of high-school football players in the USA had nasal colonization with methicillin-susceptible 
Table 4 Distribution of the SA clones within the Selected Teams

\begin{tabular}{|c|c|c|c|}
\hline Team & SA Team Carriage & Clone & Number of Strains \\
\hline \multirow[t]{5}{*}{ B004 } & $100 \%(6 / 6)$ & & \\
\hline & & A & 2 \\
\hline & & B & 2 \\
\hline & & $\mathrm{D}$ & 1 \\
\hline & & D1 & 1 \\
\hline \multirow[t]{8}{*}{$\mathrm{LOO1}^{\mathrm{a}}$} & $100 \%(11 / 11)$ & & \\
\hline & & A & 1 \\
\hline & & $\mathrm{A} 1$ & 1 \\
\hline & & C & 5 \\
\hline & & $\mathrm{C} 1$ & 1 \\
\hline & & $\mathrm{C} 3$ & 1 \\
\hline & & D & 2 \\
\hline & & $\mathrm{F}$ & 3 \\
\hline \multirow[t]{13}{*}{ R012 } & $71.8 \%(28 / 39)$ & & \\
\hline & & A & 5 \\
\hline & & $\mathrm{A} 1$ & 6 \\
\hline & & $\mathrm{A} 3$ & 1 \\
\hline & & A4 & 1 \\
\hline & & B & 1 \\
\hline & & B1 & 4 \\
\hline & & C & 2 \\
\hline & & $\mathrm{C} 2$ & 2 \\
\hline & & $\mathrm{C} 4$ & 1 \\
\hline & & $\mathrm{D}$ & 2 \\
\hline & & E & 2 \\
\hline & & E1 & 1 \\
\hline \multirow[t]{7}{*}{$\mathrm{R} 031^{\mathrm{a}}$} & $58.8 \%(10 / 17)$ & & \\
\hline & & A & 2 \\
\hline & & $\mathrm{A} 2$ & 1 \\
\hline & & B & 3 \\
\hline & & B1 & 1 \\
\hline & & $\mathrm{C} 2$ & 3 \\
\hline & & E & 1 \\
\hline
\end{tabular}

${ }^{\mathrm{a} S o m e}$ athletes carried several strains; thus, we report teams with a number of studied strains higher than the number of SA carriers

B004 was a baseball team, L001 was a wrestling team and R012 and R031 were rugby teams

SA, and none with MRSA [24]. On contrary, another study reported nose and skin MRSA carriage in $35.0 \%$ of 233 healthy athletes in the USA, and found that most isolates also harbored the PVL-encoding gene [16]. When our study started, most published data on MRSA carriage in the general and athlete populations came from the USA. A European study published during our study showed an MRSA carriage of $0.4 \%$ in the French general population [15], close to the $0.3 \%$ observed here in French athletes.

The link between SA carriage and outbreaks of skin and soft-tissue infections is not always clear. Outbreaks of PVL-positive MRSA infection have been reported in sports teams with high carriage of methicillin-susceptible SA (23.0 to $48.5 \%$ ) but not significantly linked to CAMRSA carriage [24-26]. In a 1-year surveillance study of MRSA nasal carriage among student athletes, colonization alone appeared insufficient to trigger outbreaks [24]. However, CA-SA infection outbreaks remain low in European athlete population compared to USA athlete population. The reported outbreaks are usually linked to poor hygiene practices and the implementation of simple hygiene measures can efficiently halt the transmission [27]. In our study, we did not find any PVL-positive MRSA carrier, nonetheless, the high SA carriage associated with skin contact during sport practice increases the risk of SA transmission between players [19] and therefore the risk of SA carriage and infection [7]. Previous study reported a higher risk of $S$. aureus carriage overtime in contact sports athletes and that efforts should focus to prevent transmission of $S$. aureus among these athletes [28]. Prevention of SA carriage and infection in that population could be achieved by simple hygiene measure promotion as skin wound disinfection and covering during practice, showering after practice and competition, using liquid soap rather than bar soap, washing clothes and equipment after practice, refraining from cosmetic body shaving, and discouraging athletes from sharing towels and personal hygiene items $[29,30]$. Our data did not allow identifying risk factors for SA carriage. We found that long-term antibiotic therapy was associated with SA carriage, but this classical risk factor was no longer significant in multivariate analysis. As French data on SA carriage among athletes were lacking, we based our sample size on American data; we therefore overestimated the carriage proportion, which partly explains the lack of statistical power. Sample size was a major limitation to the study. Despite showing a high carriage, it was only out of 300 athletes. Finally, one interesting control would be to apply the same swabbing technique to a sample of the Limousin general population.

We sampled our athletes only once, mainly during the first half of the sports season. Two previous studies showed variations in MRSA carriage according to the athletic activity. In a one-year surveillance study of student football and lacrosse athletes, MRSA carriage ranged from 4.0 to $23.0 \%$, with a peak during the period of maximal athletic activity [31]. Moreover, a 3-year surveillance study of CA-MRSA skin and soft-tissue infections in a collegiate football team, obtained evidence that variations in MRSA carriage might be related to the 
time of the competitive season or to outbreaks or isolated cases of CA-MRSA infection [32].

\section{Conclusions}

In our population of French athletes, we found a high SA carriage but a low MRSA carriage. Our local epidemiology was quite different than that reported in similar types of athletes in the USA, e.g., that the USA300 clone had not spread (yet) spread among our test population. We found a very high percentage of throat-only positive cultures, which substantially inflated the nasal carriage proportion. Further studies on S. aureus carriage should include throat sampling.

Staphylococcal carriage prevention in that population could consist in simple hygiene measures promotion, known to efficiently halt SA transmission in sports team.

\section{Abbreviations}

CA-MRSA: Community-associated methicillin-resistant Staphylococcus aureus: MLST: Multi locus sequence typing; MRSA: Methicillin-resistant Staphylococcus aureus; PVL: Panton Valentine leukocidin; SA: Staphylococcus aureus; ST: Sequence type

\section{Acknowledgements}

We thank Caroline Fenerol and Mathilde Saugeras for their assistance in patient inclusions and data collection, Julie Vignaud for her technical assistance and Marie Raymondeau for her help in data management. This study was sponsored by Limoges University hospital.

\section{Funding}

This study was supported by a grant from the Direction de la Recherche et Innovation de CHU de Limoges "2011" and Caisse Primaire d'Assurance Maladie de la Haute-Vienne.

\section{Availability of Data and Materials}

The datasets supporting the conclusions of this article are included within the article.

\section{Authors' Contributions}

ECD designed the research, conducted it and compiled the data to produce the submitted manuscript. DP as Clinical Investigation Center delegate doctor conducted the research and revised the manuscript. OB was in charge of the medical validation of the microbiological analysis of the research and revised the manuscript. CD supervised and participated in the conduction of the research and revised the manuscript. DC was responsible for the technical aspect of the microbiological analysis, data entry, and revised the manuscript. AL was responsible of the data managing and analysis for the research and wrote part of the manucript. NP participated in the design of the study, data interpretation and the writing of the manuscript. PMP was in charge of the statistical methodology design, analysis validation, and revised the manuscript. BF as coordinator of the Clinical Investigation Center managed the conduction of the research and revised the manuscript. MCP as Principal Investigator of the research designed, conducted the research, and produced the submitted manuscript. Each author participated sufficiently in the work to take public responsibility for appropriate portions of the content; and agreed to be accountable for all aspects of the work in ensuring that questions related to the accuracy or integrity of any part of the work are appropriately investigated and resolved.

\section{Ethics Approval and Consent to Participate}

The study, as biomedical research, was authorized by the French competent authorities and the local ethics committee (CPP). The study was carried out exactly as described in the approved protocol (Clinicaltrials.gov, NCT01148485). Each participant signed informed consent forms. The authors assert that all procedures contributing to this work comply with the ethical standards of the relevant national and institutional committees on human experimentation and with the Helsinki Declaration of 1975, as revised in 2008.

Consent for Publication

Not applicable.

\section{Competing Interests}

Elodie Couvé-Deacon, Déborah Postil, Olivier Barraud, Cécile Duchiron, Delphine Chainier, Anaïs Labrunie, Nathalie Pestourie, Pierre-Marie Preux, Bruno François, and Marie-Cécile Ploy declare that they have no conflict of interest.

\section{Author details}

${ }^{1}$ University Limoges, UMR, 1092 Limoges, France. ${ }^{2}$ INSERM, UMR, 1092 Limoges, France. ${ }^{3} \mathrm{CHU}$ Limoges, Laboratory of

Bacteriology-Virology-Hygiene, 2 avenue Martin Luther King, 87042 Limoges, cedex, France. ${ }^{4}$ INSERM, CIC-1435, F-87000 Limoges, France. ${ }^{5} \mathrm{CHU}$ Limoges, Centre d'Epidémiologie de Biostatistique et de Méthodologie de la Recherche, Limoges, France.

Received: 24 March 2017 Accepted: 18 July 2017

Published online: 16 August 2017

\section{References}

1. Dufour P, Gillet $Y$, Bes M, Lina G, Vandenesch F, Floret D, Etienne J, Richet H. Community-acquired methicillin-resistant Staphylococcus aureus infections in France: emergence of a single clone that produces Panton-Valentine leukocidin. Clin Infect Dis. 2002;35:819-24.

2. Otter JA, French GL. Community-associated meticillin-resistant Staphylococcus aureus strains as a cause of healthcare-associated infection. J Hosp Infect. 2011;79:189-93.

3. Otter JA, French GL. Molecular epidemiology of community-associated meticillin-resistant Staphylococcus aureus in Europe. Lancet Infect Dis. 2010; 10:227-39.

4. Tenover FC, Goering RV. Methicillin-resistant Staphylococcus aureus strain USA300: origin and epidemiology. J Antimicrob Chemother. 2009:64:441-6.

5. Cohen PR. The skin in the gym: a comprehensive review of the cutaneous manifestations of community-acquired methicillin-resistant Staphylococcus aureus infection in athletes. Clin Dermatol. 2008;26:16-26.

6. Grosset-Janin A, Nicolas $X$, Saraux A. Sport and infectious risk: a systematic review of the literature over 20 years. Med Mal Infect. 2012;42:533-44.

7. Wertheim HFL, Melles DC, Vos MC, van Leeuwen W, van Belkum A, Verbrugh HA, Nouwen JL. The role of nasal carriage in Staphylococcus aureus infections. Lancet Infect Dis. 2005;5:751-62.

8. Kluytmans J, van Belkum A, Verbrugh H. Nasal carriage of Staphylococcus aureus: epidemiology, underlying mechanisms, and associated risks. Clin Microbiol Rev. 1997:10:505-20.

9. Beam JW, Buckley B. Community-acquired methicillin-resistant Staphylococcus aureus: prevalence and risk factors. J Athl Train. 2006;41:337-40.

10. Salgado CD, Farr BM, Calfee DP. Community-acquired methicillin-resistant Staphylococcus aureus: a meta-analysis of prevalence and risk factors. Clin Infect Dis. 2003;36:131-9.

11. Lem P, Spiegelman J, Toye B, Ramotar K. Direct detection of mecA, nuc and $16 \mathrm{~S}$ rRNA genes in BacT/Alert blood culture bottles. Diagn Microbiol Infect Dis. 2001;41:165-8.

12. Jarraud S, Mougel C, Thioulouse J, Lina G, Meugnier H, Forey F, Nesme X, Etienne J, Vandenesch F. Relationships between Staphylococcus aureus genetic background, virulence factors, agr groups (alleles), and human disease. Infect Immun. 2002;70:631-41.

13. Enright MC, Day NP, Davies CE, Peacock SJ, Spratt BG. Multilocus sequence typing for characterization of methicillin-resistant and methicillin-susceptible clones of Staphylococcus aureus. J Clin Microbiol. 2000;38:1008-15.

14. Vázquez-Sánchez D, López-Cabo M, Saá-Ibusquiza P, Rodríguez-Herrera JJ. Incidence and characterization of Staphylococcus aureus in fishery products marketed in Galicia (Northwest Spain). Int J Food Microbiol. 2012:157:286-96.

15. den Heijer CDJ, van Bijnen EME, Paget WJ, Pringle M, Goossens $H$, Bruggeman CA, Schellevis FG, Stobberingh EE. APRES Study Team. Prevalence and resistance of commensal Staphylococcus aureus, including meticillin-resistant S aureus, in nine European countries: a cross-sectional study. Lancet Infect Dis. 2013;13:409-15. 
16. Champion AE, Goodwin TA, Brolinson PG, Werre SR, Prater MR, Inzana TJ. Prevalence and characterization of methicillin-resistant Staphylococcus aureus isolates from healthy university student athletes. Ann Clin Microbiol Antimicrob. 2014;13:33.

17. Schechter-Perkins EM, Mitchell PM, Murray KA, Rubin-Smith JE, Weir S, Gupta K. Prevalence and predictors of nasal and extranasal staphylococcal colonization in patients presenting to the emergency department. Ann Emerg Med. 2011;57:492-9.

18. Sollid JU, Furberg AS, Hanssen AM, Johannessen M. Staphylococcus aureus: determinants of human carriage. Infect Genet Evol. 2014;21:531-41.

19. Suzuki K, Tagami K. Role of nasal Staphylococcus aureus carriage in transmission among contact athletes. Int J Sports Med. 2015;36:1186-91.

20. Fall C, Richard V, Dufougeray A, Biron A, Seck A, Laurent F, Breurec S. Staphylococcus aureus nasal and pharyngeal carriage in Senegal. Clin Microbiol Infect. 2014:20:0239-41.

21. Van Nguyen $K$, Zhang T, Thi Vu BN, Dao TT, Tran TK, Thi Nguyen DN, Thi Tran HK, Thi Nguyen CK, Fox A, Horby P, Wertheim H. Staphylococcus aureus nasopharyngeal carriage in rural and urban northern Vietnam. Trans R Soc Trop Med Hyg. 2014;108:783-90.

22. Rackham DM, Ray SM, Franks AS, Bielak KM, Pinn TM. Community-associated methicillin-resistant Staphylococcus aureus nasal carriage in a college student athlete population. Clin J Sport Med. 2010;20:185-8.

23. Dauwalder O, Lina G, Durand G, Bes M, Meugnier H, Jarlier V, Coignard B, Vandenesch F, Etienne J, Laurent F. Epidemiology of invasive methicillinresistant Staphylococcus aureus clones collected in France in 2006 and 2007. J Clin Microbiol. 2008;46:3454-8.

24. Lear A, McCord G, Peiffer J, Watkins RR, Parikh A, Warrington S. Incidence of Staphylococcus aureus nasal colonization and soft tissue infection among high school football players. J Am Board Fam Med. 2011;24:429-35.

25. Begier EM, Frenette K, Barrett NL, Mshar P, Petit S, Boxrud DJ, WatkinsColwell K, Wheeler S, Cebelinski EA, Glennen A, Nguyen D, Hadler JL, Connecticut Bioterrorism Field Epidemiology Response Team. A high-morbidity outbreak of methicillin-resistant Staphylococcus aureus among players on a college football team, facilitated by cosmetic body shaving and turf burns. Clin Infect Dis. 2004;39:1446-53.

26. Kazakova SV, Hageman JC, Matava M, Srinivasan A, Phelan L, Garfinkel B, Boo T, McAllister S, Anderson J, Jensen B, Dodson D, Lonsway D, McDougal LK, Arduino M, Fraser VJ, Killgore G, Tenover FC, Cody S, Jernigan DB. A clone of methicillin-resistant Staphylococcus aureus among professional football players. N Engl J Med. 2005;352:468-75.

27. Couvé-Deacon E, Tristan A, Pestourie N, Faure C, Doffoel-Hantz V, Garnier F, Laurent F, Lina G, Ploy M-C. Outbreak of Panton-Valentine leukocidinassociated methicillin-susceptible Staphylococcus aureus infection in a rugby team, France, 2010-2011. Emerg Infect Dis. 2016;22:96-9.

28. Jiménez-Truque N, Saye EJ, Soper N, Saville BR, Thomsen I, Edwards KM, Creech CB. Association between contact sports and colonization with Staphylococcus aureus in a prospective cohort of collegiate athletes. Sports Med. 2016; doi:10.1007/s40279-016-0618-6

29. Levine H, Kayouf R, Rozhavski V, Sela T, Rajuan-Galor I, Ferber AT, Yona S, Gorochovski O, Halperin T, Hartal M. Neglect of skin wounds and the risk of becoming a Staphylococcus aureus nasal carrier: a cohort study. BMC Public Health. 2015;15:749.

30. Zinder SM, Basler RSW, Foley J, Scarlata C, Vasily DB. National Athletic Trainers' Association Position Statement: Skin Diseases. J Athl Train. 2010;45:411-28.

31. Creech CB, Saye E, McKenna BD, Johnson BG, Jimenez N, Talbot TR, Bossung T, Gregory A, Edwards KM. One-year surveillance of methicillin-resistant Staphylococcus aureus nasal colonization and skin and soft tissue infections in collegiate athletes. Arch Pediatr Adolesc Med. 2010;164:615-20.

32. Romano R, Lu D, Holtom P. Outbreak of community-acquired methicillinresistant Staphylococcus aureus skin infections among a collegiate football team. J Athl Train. 2006;41:141-5.

\section{Submit your manuscript to a SpringerOpen ${ }^{\odot}$ journal and benefit from:}

- Convenient online submission

- Rigorous peer review

- Open access: articles freely available online

- High visibility within the field

- Retaining the copyright to your article

Submit your next manuscript at $\gg$ springeropen.com 